\title{
Systematic Review \\ Dietary Fibre Intake in Relation to Asthma, Rhinitis and Lung Function Impairment-A Systematic Review of Observational Studies
}

\author{
Emmanouela Sdona ${ }^{1, *(D)}$, Athina Vasiliki Georgakou ${ }^{1}\left(\mathbb{D}\right.$, Sandra Ekström ${ }^{1,2}$ and Anna Bergström ${ }^{1,2}$ \\ 1 Institute of Environmental Medicine, Karolinska Institute, 17177 Stockholm, Sweden; \\ athina.georgakou@ki.se (A.V.G.); sandra.ekstrom@ki.se (S.E.); anna.bergstrom@ki.se (A.B.) \\ 2 Centre for Occupational and Environmental Medicine, Region Stockholm, 11365 Stockholm, Sweden \\ * Correspondence: emmanouela.sdona@ki.se
}

check for

updates

Citation: Sdona, E.; Georgakou, A.V.; Ekström, S.; Bergström, A. Dietary Fibre Intake in Relation to Asthma, Rhinitis and Lung Function

Impairment-A Systematic Review of Observational Studies. Nutrients 2021 13, 3594. https://doi.org/10.3390/ nu13103594

Academic Editor: Linette E.M.

Willemsen

Received: 22 September 2021

Accepted: 11 October 2021

Published: 14 October 2021

Publisher's Note: MDPI stays neutral with regard to jurisdictional claims in published maps and institutional affiliations.

Copyright: (c) 2021 by the authors. Licensee MDPI, Basel, Switzerland. This article is an open access article distributed under the terms and conditions of the Creative Commons Attribution (CC BY) license (https:/ / creativecommons.org/licenses/by/ $4.0 /)$.

\begin{abstract}
A high intake of dietary fibre has been associated with a reduced risk of several chronic diseases. This study aimed to review the current evidence on dietary fibre in relation to asthma, rhinitis and lung function impairment. Electronic databases were searched in June 2021 for studies on the association between dietary fibre and asthma, rhinitis, chronic obstructive pulmonary disease (COPD) and lung function. Observational studies with cross-sectional, case-control or prospective designs were included. Studies on animals, case studies and intervention studies were excluded. The quality of the evidence from individual studies was evaluated using the RoB-NObs tool. The World Cancer Research Fund criteria were used to grade the strength of the evidence. Twenty studies were included in this systematic review, of which ten were cohort studies, eight cross-sectional and two case-control studies. Fibre intake during pregnancy or childhood was examined in three studies, while seventeen studies examined the intake during adulthood. There was probable evidence for an inverse association between dietary fibre and COPD and suggestive evidence for a positive association with lung function. However, the evidence regarding asthma and rhinitis was limited and inconsistent. Further research is needed on dietary fibre intake and asthma, rhinitis and lung function among adults and children.
\end{abstract}

Keywords: diet; fibres; asthma; rhinitis; respiratory test; obstructive lung disease

\section{Introduction}

Epidemiologic evidence has consistently shown that a high intake of dietary fibre is associated with a reduced risk of several chronic diseases, such as cardiovascular diseases, cancer, type 2 diabetes and obesity, as well as of total and specific-cause mortality [1-3]. Fibre-rich, plant-based dietary patterns, including grains, fruits, vegetables and nuts, stimulate the growth of beneficial bacterial species and contribute to a healthy colonic microbiota ecosystem due to the fermentation of fibres into short-chain fatty acids (SCFAs) [4].

Asthma is a chronic inflammatory disorder of the airways and the most common chronic disease among children. It is a cause of substantial burden of a disease, including a reduced quality of life in people of all ages and premature death [5]. Children with asthma, particularly those with persistent and severe forms of asthma, may attain a lower maximum lung function in adulthood, which increases the risk for the development of chronic obstructive pulmonary disease (COPD) [6]. Additionally, asthma frequently coexists with rhinitis, mostly among adolescents, as well as other atopic diseases, and it has been suggested that allergy-related diseases cannot be studied as isolated entities [7]. Both genetic and environmental factors have been implicated in the aetiology of the aforementioned diseases; however, the increase in the prevalence of asthma and other allergic diseases in the second half of the 20th century has been mostly associated with environmental factors, such as smoking, air pollution and changes in lifestyle and diet [8]. 
Following this increase, an increasing interest in identifying potentially modifiable factors has been expressed in the literature.

In recent years, epidemiological studies have also explored the association between dietary fibres and respiratory and allergic diseases. Dietary fibres may influence the development of respiratory and atopic outcomes through different mechanisms-for example, through the antioxidant and anti-inflammatory effects of whole grains, by enhancing the bio-accessibility of antioxidants from fruits and vegetables or through immunomodulatory effects induced by changes in the gut microbiota [9-11]. However, the epidemiological evidence for this potential association has not been systematically reviewed.

The aim of this systematic review is, therefore, to explore the existing evidence on dietary fibre intake in relation to asthma, rhinitis, COPD and lung function.

\section{Materials and Methods}

\subsection{Protocol and Registration}

This systematic review was performed according to the PRISMA guidelines [12] (checklist in the Supplementary Materials), and an application for registration in PROSPERO was submitted.

\subsection{Eligibility Criteria}

Original studies reporting empirical findings on the association between dietary fibre intake and at least one outcome of interest (asthma; rhinitis; COPD, or symptoms of the aforementioned diseases, such as wheeze, cough, and phlegm; lung function) were searched. Observational studies on humans with cross-sectional, case-control or prospective designs were included. Studies on animals, case studies (case reports or case series) and intervention studies were excluded.

\subsection{Information Sources}

Systematic searches using predefined search terms were performed in multiple databases, including Medline (OVID), Embase, Cochrane Library, Web of Science and Scopus. The databases were searched from inception, limited to the English, French, German and Swedish languages. Additionally, reference lists of the articles included in the review and of relevant review studies were manually screened to identify other relevant articles. Information from conference abstracts, dissertations and grey literature (e.g., reports) was not included.

\subsection{Search Strategy}

The search was conducted in June 2021 based on the term construct used for Medline (see the Supplementary Materials), assisted by professional librarians at the Karolinska Institute University Library. The following MeSH terms were used in the Medline (OVID) search: Dietary Fiber, Lung Diseases, Obstructive, Rhinitis and Respiratory Function Tests. The MeSH terms were adapted in accordance with the corresponding vocabulary in Embase Emtree. Each concept was also complemented with relevant free-text terms. The free-text terms were, if appropriate, truncated and/or combined with proximity operators. The full search strategies are available in the Supplementary Materials.

\subsection{Study Selection}

The search results were exported to Endnote X9, where duplicates were excluded. As the first step, relevant articles were considered based on their title and abstract. At the second step, full-text versions of the selected papers were examined. In case there were multiple publications from the same cohort study, they were all included if they referred to different outcomes of interest. Following the above inclusion and exclusion criteria, two reviewers (E.S. and A.V.G.), without consideration for the results, performed the assessment of the studies for potential inclusion independently. Any differences in 
opinions were resolved through discussion until a consensus was reached. A third reviewer (S.E.) was consulted when necessary.

\subsection{Data Extraction}

The two reviewers independently conducted the data extraction from each study using a predefined data extraction sheet. The items extracted regarding the study characteristics comprised the first author name; year of publication; objectives; country; name of cohort (if applicable); study design; sample size; source population (age, sex and other characteristics); exposure assessment; categorisation of exposure; outcome assessment; mean follow-up period (if applicable); statistical methods; effect measures; covariates; missing data; control for selection bias and confounding, effect modifications and sensitivity analyses.

\subsection{Risk of Bias in Individual Studies}

The 'Risk of Bias for Nutrition Observational Studies' (RoB-NObS) tool recently developed by the US Department of Agriculture (USDA) Nutrition Evidence Systematic Review (NESR) team [13] was used to assess the risk of bias in individual studies independently by two reviewers (E.S. and A.V.G.) in the following domains: bias due to confounding, selection bias, bias in the classification of exposures, bias due to departures from intended exposures, bias due to missing data, bias in the measurement of outcomes and bias in the selection of reported results. On the occasion of discrepancies, a third reviewer (S.E.) assessed the study, and a consensus was achieved.

\subsection{Presentation/Synthesis of Results}

We performed a qualitative synthesis of the results, including a summary table presenting the association between the total fibre intake and the outcomes of interest (highest vs. lowest category and $p$ for trends) from each study. Associations between different sources of fibre and the outcomes, as well as the stratified analysis results, are reported in text only.

\subsection{Risk of Bias across Studies}

The World Cancer Research Fund (WCRF) criteria [14], applied as suggested by Arnesen et al. [15], were used to grade the strength of the evidence for each outcome of interest as convincing (high), probable (moderate), limited/suggestive (low) and limited/no conclusion (insufficient).

\section{Results}

\subsection{Study Selection}

A flowchart of the study selection is presented in Figure 1. Briefly, the search of the electronic databases yielded 1328 articles, 169 of which were considered relevant after title and abstract screening. Additionally, one article was identified in the reference lists. Finally, 20 articles were considered for inclusion in the systematic review. Of these, ten were cohort studies, eight cross-sectional studies and two case-control studies. Ten studies were published the last five years (2017-2021) and ten studies before 2017 (2004-2016). Seven studies were conducted in the US, seven studies in Asia, three studies in Europe and three studies in Australia. 
Identification of studies

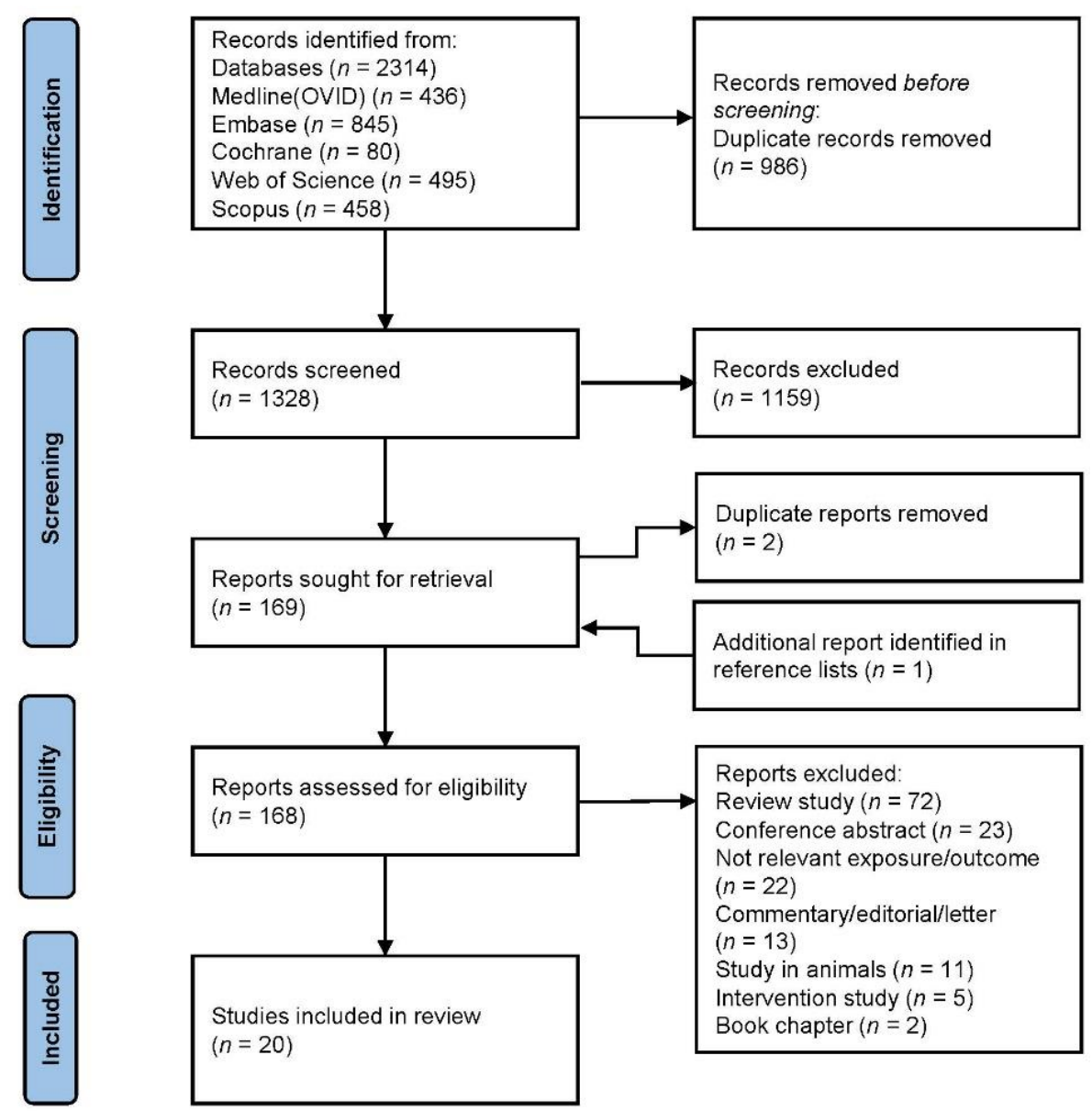

Figure 1. PRISMA flowchart of the study selection.

\subsection{Study Characteristics}

One birth cohort study explored fibre intake during pregnancy [16], two studies explored fibre intake during childhood $[17,18]$ and 17 studies explored fibre intake during adulthood [19-35] (Table 1). Sixteen studies were population-based [18-29,31-34], while four studies were conducted on high-risk populations (infants with a family history of allergic disease [16], migrant adolescents [17], COPD adults [30] or smokers [35]). Half of the studies included fibre intake as the primary exposure $[16,19,20,22,24,26-29,32]$, while the other half studied fibre intake as part of the nutrient or food intake or dietary pattern $[17,18,21,23,25,30,31,33-35]$. With regards to the outcomes of interest, seven studies reported associations with asthma/asthma symptoms [16-20,22,23], two studies with rhinitis [20,21], eight studies with COPD/COPD symptoms [24-31] and six studies with lung function $[23,24,32-35]$ (seven studies reported more than one outcome). 
Table 1. Characteristics of the studies examining the association between dietary fibre intake and asthma, rhinitis, COPD, and lung function.

\begin{tabular}{|c|c|c|c|c|c|c|c|c|c|}
\hline $\begin{array}{l}\text { Author, } \\
\text { Year, } \\
\text { Country, } \\
\text { Cohort }\end{array}$ & $\begin{array}{l}\text { Study } \\
\text { Design }\end{array}$ & Sample Size & Population & $\begin{array}{l}\text { Exposure } \\
\text { Assessment }\end{array}$ & $\begin{array}{l}\text { Outcome } \\
\text { Assessment }\end{array}$ & Follow-Up & $\begin{array}{l}\text { Statistical } \\
\text { Methods }\end{array}$ & Effect Measures & Covariates \\
\hline \multicolumn{10}{|c|}{ Maternal fibre intake during pregnancy } \\
\hline $\begin{array}{c}\text { Pretorius et al., } \\
\text { 2019, } \\
\text { Australia [16] }\end{array}$ & $\mathrm{C}$ & $\begin{array}{c}639 \\
\text { mother-infant } \\
\text { pairs }\end{array}$ & $\begin{array}{c}\text { mothers } \\
\text { aged } \geq 18 \text { years, } \\
\text { non-smokers, } \\
\text { infants with family } \\
\text { history of } \\
\text { allergic disease }\end{array}$ & $\begin{array}{l}\text { semi-quantitative } \\
\text { 101-item FFQ at } \\
36-40 \text { weeks' } \\
\text { gestation } \\
\text { assessing intake } \\
\text { over one month }\end{array}$ & $\begin{array}{c}\text { parent reported } \\
\text { and doctor } \\
\text { diagnosed wheeze }\end{array}$ & 12 months & $\begin{array}{l}\text { logistic } \\
\text { regression, } \\
\text { multinomial } \\
\text { logistic } \\
\text { regression }\end{array}$ & $\begin{array}{c}\text { total fibre (g/day), median } 23.8 \\
\text { OR }(95 \% \mathrm{CI}) 0.99(0.99-1.00) \\
\text { parent reported wheeze } \\
\text { OR }(95 \% \text { CI) } 0.98(0.94-1.01) \\
\text { doctor diagnosed wheeze }\end{array}$ & $\begin{array}{c}\text { maternal age, education, } \\
\text { ethnicity, child's gender, } \\
\text { birth weight, gestational } \\
\text { age at birth, pet ownership, } \\
\text { maternal parity, } \\
\text { delivery mode }\end{array}$ \\
\hline \multicolumn{10}{|c|}{ Fibre intake during childhood } \\
\hline $\begin{array}{l}\text { Wood et al., } \\
\text { 2015, } \\
\text { Australia [17] }\end{array}$ & CS & 144 & $\begin{array}{l}\text { adolescents aged } \\
12-18 \text { years }\end{array}$ & $\begin{array}{l}\text { interviewer } \\
\text { administered } \\
\text { 107-item FFQ }\end{array}$ & $\begin{array}{l}\text { ISAAC video } \\
\text { questionnaire, } \\
\text { spirometry }\end{array}$ & - & $\begin{array}{l}\text { logistic } \\
\text { regression }\end{array}$ & $\begin{array}{c}\text { total fibre }(\mathrm{g} / \text { day }) \\
\text { OR }(95 \% \mathrm{CI}) 1.0(1.0-1.0) \\
\text { self-reported wheeze } \\
\text { energy adjusted fibre, median } \\
6.7 \mathrm{~g} / 1000 \mathrm{kcal}\end{array}$ & $\begin{array}{l}\text { age, sex, length of time } \\
\text { in Australia }\end{array}$ \\
\hline $\begin{array}{c}\text { Vaccaro et al., } \\
\text { 2016, US, } \\
\text { NHANES [18] }\end{array}$ & CS & 4133 & $\begin{array}{l}\text { children aged } \\
2-11 \text { years }\end{array}$ & $24 \mathrm{~h}$ dietary recall & $\begin{array}{l}\text { self-reported } \\
\text { asthma }\end{array}$ & - & $\begin{array}{l}\text { logistic } \\
\text { regression }\end{array}$ & $\begin{array}{c}\text { Q1 vs. Q4 OR (95\% CI) } 1.31 \\
(0.88-1.96), p \text {-trend } 0.034 \text { ever } \\
\text { asthma, Q1 vs. Q4 OR }(95 \% \text { CI) } \\
1.38(0.87-2.20), p \text {-trend } 0.027 \\
\text { current asthma }\end{array}$ & $\begin{array}{l}\text { age, gender, second-hand } \\
\text { smoke exposure, income }\end{array}$ \\
\hline \multicolumn{10}{|c|}{$\begin{array}{l}\text { Fibre intake during adulthood } \\
\text { Asthma, rhinitis and related symptoms }\end{array}$} \\
\hline $\begin{array}{c}\text { Saeed et al., } \\
\text { 2020, US, } \\
\text { NHANES [19] }\end{array}$ & CS & 13,147 & $\begin{array}{c}\text { adults aged } \\
\text { 20-79 years } \\
\text { (mean age } 46 \text { years) }\end{array}$ & $\begin{array}{l}\text { two-interviewer- } \\
\text { administered } \\
24 \mathrm{~h} \text { recalls }\end{array}$ & $\begin{array}{l}\text { self-reported } \\
\text { asthma, wheeze, } \\
\text { cough, phlegm } \\
\text { production, } \\
\text { blood CRP }\end{array}$ & - & $\begin{array}{l}\text { logistic } \\
\text { regression, } \\
\text { multinomial } \\
\text { logistic } \\
\text { regression }\end{array}$ & $\begin{array}{c}\text { total fibre } \mathrm{Q} 1 \text { vs. Q4 } \\
(<10.5 \text { vs. }>21.2 \mathrm{~g} / \text { day }) \\
\text { OR }(95 \% \mathrm{CI}) 1.4(1.0-1.8), \\
p \text {-trend } 0.092 \text { asthma, OR } \\
(95 \% \text { CI }) 1.3(1.0-1.6), p \text {-trend } \\
0.017 \text { wheeze, OR }(95 \% \mathrm{CI}) 1.7 \\
(1.2-2.3), p \text {-trend } 0.0003 \text { cough, } \\
\text { OR }(95 \% \mathrm{CI}) 1.4(1.1-2.0), \\
p \text {-trend } 0.011 \text { phlegm }\end{array}$ & $\begin{array}{c}\text { age, race/ethnicity, sex, } \\
\text { smoking status, BMI, } \\
\text { poverty index ratio, total } \\
\text { energy intake }\end{array}$ \\
\hline $\begin{array}{l}\text { Lee et al., 2021, } \\
\text { South Korea, } \\
\text { Korean } \\
\text { NHANES [20] }\end{array}$ & CS & 10,479 & $\begin{array}{c}\text { adults aged } 19 \text { years } \\
\text { and older } \\
\text { (mean age } 51 \text { years) }\end{array}$ & 63-item FFQ & $\begin{array}{c}\text { self-reported } \\
\text { asthma, } \\
\text { self-reported } \\
\text { rhinitis plus nasal } \\
\text { endoscopy, serum } \\
\text { IgE and specific } \\
\text { IgE levels }\end{array}$ & - & $\begin{array}{l}\text { logistic } \\
\text { regression }\end{array}$ & $\begin{array}{c}\text { total fibre Q4 vs. Q1 } \\
\text { OR }(95 \% \mathrm{CI}) 0.66(0.48-0.91) \\
p \text {-trend }<0.001 \text { asthma, } \\
\text { OR }(95 \% \mathrm{CI}) 0.95(0.77-1.17) \\
p \text {-trend }<0.001 \text { allergic rhinitis }\end{array}$ & $\begin{array}{l}\text { age, sex, household } \\
\text { income, residency, alcohol } \\
\text { consumption, smoking } \\
\text { status, BMI, physical } \\
\text { activity, other nutrients }\end{array}$ \\
\hline
\end{tabular}


Table 1. Cont.

\begin{tabular}{|c|c|c|c|c|c|c|c|c|c|}
\hline $\begin{array}{l}\text { Author, } \\
\text { Year, } \\
\text { Country, } \\
\text { Cohort }\end{array}$ & $\begin{array}{l}\text { Study } \\
\text { Design }\end{array}$ & Sample Size & Population & $\begin{array}{l}\text { Exposure } \\
\text { Assessment }\end{array}$ & $\begin{array}{l}\text { Outcome } \\
\text { Assessment }\end{array}$ & Follow-Up & $\begin{array}{l}\text { Statistical } \\
\text { Methods }\end{array}$ & Effect Measures & Covariates \\
\hline \multicolumn{10}{|c|}{$\begin{array}{l}\text { Fibre intake during adulthood } \\
\text { Asthma, rhinitis and related symptoms }\end{array}$} \\
\hline $\begin{array}{l}\text { Miyake et al., } \\
\text { 2006, } \\
\text { Japan, } \\
\text { Osaka Maternal } \\
\text { and Child } \\
\text { Health } \\
\text { Study [21] }\end{array}$ & CS & 1002 & pregnant women & $\begin{array}{l}\text { 147-item } \\
\text { questionnaire } \\
\text { assessing intake } \\
\text { over one month }\end{array}$ & $\begin{array}{l}\text { allergic rhinitis } \\
\text { drug treatment } \\
\text { during the } \\
\text { previous } \\
12 \text { months }\end{array}$ & - & $\begin{array}{l}\text { logistic } \\
\text { regression }\end{array}$ & $\begin{array}{c}\text { energy adjusted fibre (g/day) } \\
\text { Q4 (14.7) vs. Q1 (8.2) } \\
\text { OR (95\% CI) } 1.14(0.66-2.00) \\
p \text {-trend } 0.80\end{array}$ & $\begin{array}{c}\text { smoking, indoor domestic } \\
\text { pets, family history of } \\
\text { asthma, eczema, rhinitis, } \\
\text { family income, education, } \\
\text { mite allergen level in } \\
\text { house dust, changes in diet } \\
\text { in the previous month, } \\
\text { season of data collection, } \\
\text { BMI }\end{array}$ \\
\hline $\begin{array}{l}\text { Andrianasolo } \\
\text { et al., 2019, } \\
\text { France, } \\
\text { NutriNet-Sante } \\
\text { Study [22] }\end{array}$ & CS & $\begin{array}{l}26,640 \text { women } \\
\text { and } 8740 \text { men }\end{array}$ & $\begin{array}{l}\text { adults aged } 18 \text { years } \\
\text { and older (mean age } \\
53 \text { years in women, } \\
59 \text { years in men) }\end{array}$ & $\begin{array}{c}\text { three } \\
\text { self-administered } \\
\text { web-based } 24 \mathrm{~h} \\
\text { dietary records }\end{array}$ & $\begin{array}{l}\text { self-reported } \\
\text { asthma symptom } \\
\text { score, asthma } \\
\text { control test }\end{array}$ & - & $\begin{array}{l}\text { binomial } \\
\text { negative } \\
\text { regression, } \\
\text { logistic } \\
\text { regression }\end{array}$ & $\begin{array}{c}\text { total fibre Q5 vs. Q1 } \\
\text { (28.6 vs. } 13.8 \mathrm{~g} / \text { day in women, } \\
30.5 \text { vs. } 12.7 \mathrm{~g} / \text { day in men) } \\
\text { asthma symptom score OR } \\
(95 \% \mathrm{CI}) 0.73(0.67-0.79) \\
p \text {-trend }<0.001 \text { in women and } \\
\text { OR }(95 \% \mathrm{CI}) 0.63(0.55-0.73) \\
\text {-trend }<0.001 \text { in men }\end{array}$ & $\begin{array}{c}\text { age, educational level, } \\
\text { smoking status, pack-years } \\
\text { of smoking, BMI, physical } \\
\text { activity, total energy } \\
\text { intake, allergic rhinitis, } \\
\text { family history of asthma }\end{array}$ \\
\hline $\begin{array}{c}\text { Berthon et al., } \\
\text { 2013, } \\
\text { Australia [23] }\end{array}$ & $\mathrm{CC}$ & $\begin{array}{l}137 \text { cases with } \\
\text { asthma (of } \\
\text { which } 64 \text { with } \\
\text { severe persistent } \\
\text { asthma), } \\
65 \text { controls }\end{array}$ & $\begin{array}{l}\text { adults aged } 18 \text { years } \\
\text { and older } \\
\text { (mean age } 53 \text { years) }\end{array}$ & $\begin{array}{c}\text { 186-item } \\
\text { semi-quantitative } \\
\text { FFQ }\end{array}$ & $\begin{array}{l}\text { asthma severity, } \\
\text { lung function } \\
\text { (eNO, spirometry, } \\
\text { sputum cells) }\end{array}$ & - & $\begin{array}{l}\text { logistic and } \\
\text { linear regression }\end{array}$ & $\begin{array}{c}\text { energy adjusted fibre (mean } \\
\text { cases } 32 \mathrm{~g} / \text { day, controls } \\
37 \mathrm{~g} / \text { day) } \\
\text { severe persistent asthma } \\
\text { OR }(95 \% \text { CI) } 0.94(0.90-0.99) \\
\text { Coefficients for dietary fibre } \\
\text { intake in asthmatics } \\
\text { FEV1 } 0.02 \mathrm{~L} \text {, FVC } 0.02 \mathrm{~L}, \\
\text { FEV1/FVC } 0.002, \text { airway } \\
\% \text { eosinophils }-0.36, \\
\% \text { neutrophils } 0.26\end{array}$ & $\begin{array}{l}\text { age, gender, BMI, } \\
\text { total energy }\end{array}$ \\
\hline
\end{tabular}


Table 1. Cont.

\begin{tabular}{|c|c|c|c|c|c|c|c|c|c|}
\hline $\begin{array}{l}\text { Author, } \\
\text { Year, } \\
\text { Country, } \\
\text { Cohort }\end{array}$ & $\begin{array}{l}\text { Study } \\
\text { Design }\end{array}$ & Sample Size & Population & $\begin{array}{l}\text { Exposure } \\
\text { Assessment }\end{array}$ & $\begin{array}{c}\text { Outcome } \\
\text { Assessment }\end{array}$ & Follow-Up & $\begin{array}{l}\text { Statistical } \\
\text { Methods }\end{array}$ & Effect Measures & Covariates \\
\hline \multicolumn{10}{|c|}{ COPD and related symptoms } \\
\hline $\begin{array}{c}\text { Kan et al., 2008, } \\
\text { US, ARIC } \\
\text { study [24] }\end{array}$ & CS & 11,897 & $\begin{array}{l}\text { adults aged } \\
44-66 \text { years }\end{array}$ & $\begin{array}{l}\text { interviewer- } \\
\text { administered } \\
66 \text {-item semi- } \\
\text { quantitative FFQ }\end{array}$ & $\begin{array}{l}\text { spirometry, COPD } \\
\text { based on } \\
\text { self-reported } \\
\text { symptoms or } \\
\text { spirometry }\end{array}$ & 3 years & $\begin{array}{l}\text { linear } \\
\text { regression, } \\
\text { logistic } \\
\text { regression }\end{array}$ & $\begin{array}{c}\text { Q5 (26.7) vs. Q1 (9.5) } \\
\text { OR (95\% CI) COPD prevalence } \\
0.85(0.68-1.05), p \text {-trend } 0.044 \\
\text { Coefficients }(95 \% \text { CI) FEV1 } \\
60.2 \mathrm{~mL}(27.7-92.7), \\
p \text {-trend }<0.001, \text { FVC } 55.2 \mathrm{~mL} \\
(18.2-92.3), p \text {-trend } 0.001, \\
\text { FEV1/FVC } 0.4(-0.1-0.9), \\
p \text {-trend } 0.040, \% \text { predicted } \\
\text { FEV1 1.8 }(0.8-2.9), \\
p \text {-trend }<0.001, \% \text { predicted } \\
\text { FVC } 1.4(0.4-2.4), p \text {-trend } 0.001\end{array}$ & $\begin{array}{l}\text { BMI, age, ethnicity, gender, } \\
\text { study centre, smoking } \\
\text { status, pack-years, } \\
\text { occupation, education, } \\
\text { diabetes status, } \\
\text { residence-based traffic } \\
\text { density, total energy } \\
\text { intake, glycaemic index, } \\
\text { micronutrients from both } \\
\text { food and supplements, } \\
\text { and cured meat }\end{array}$ \\
\hline $\begin{array}{l}\text { Hirayama et al., } \\
\text { 2009, Japan [25] }\end{array}$ & $\mathrm{CC}$ & $\begin{array}{l}278 \text { cases with } \\
\text { COPD, } \\
340 \text { controls }\end{array}$ & $\begin{array}{c}\text { adults aged } \\
50-75 \text { years } \\
\text { (mean age 66 years) }\end{array}$ & $\begin{array}{l}\text { 138-item FFQ } \\
\text { assessing intake } \\
\text { over the previous } \\
\text { five years }\end{array}$ & $\begin{array}{l}\text { spirometry } \\
\text { diagnosed COPD } \\
\text { within the past } \\
\text { four years }\end{array}$ & - & $\begin{array}{l}\text { unconditional } \\
\text { logistic } \\
\text { regression }\end{array}$ & $\begin{array}{c}\text { total fibre }(\mathrm{g} / \text { day) } \mathrm{Q} 4(\geq 16.08) \\
\text { vs. Q1 }(\leq 8.84) \\
\text { OR }(95 \% \text { CI) } 0.49(0.26-0.95) \\
p \text {-trend } 0.160\end{array}$ & $\begin{array}{c}\text { age, gender, BMI, } \\
\text { education level, life-long } \\
\text { physical activity } \\
\text { involvement, smoking } \\
\text { status, smoking } \\
\text { pack-years, alcohol } \\
\text { drinking, intake of fish, } \\
\text { red meat and chicken, total } \\
\text { energy intake }\end{array}$ \\
\hline $\begin{array}{l}\text { Butler et al., } \\
\text { 2004, Singapore, } \\
\text { Singapore } \\
\text { Chinese Health } \\
\text { Study [26] }\end{array}$ & C & 49,140 & $\begin{array}{l}\text { adults aged } \\
45-74 \text { years of } \\
\text { Chinese origin }\end{array}$ & $\begin{array}{c}165 \text {-item } \\
\text { quantitative FFQ }\end{array}$ & $\begin{array}{l}\text { self-reported } \\
\text { incident cough } \\
\text { with phlegm }\end{array}$ & 5.3 years & $\begin{array}{l}\text { unconditional } \\
\text { logistic } \\
\text { regression }\end{array}$ & $\begin{array}{c}\text { Q4 }(11.6) \text { vs. Q1 }(4.7) \\
\text { OR }(95 \% \text { CI }) \text { non-starch } \\
\text { polysaccharides }(\mathrm{g}) \\
0.61(0.47-0.78), p \text {-trend } 0.001, \\
\text { fruit } 0.67(0.52-0.87), p \text {-trend } \\
0.006, \text { grain } 1.12(0.80-1.56), \\
p \text {-trend } 0.301, \text { vegetable } 0.92 \\
(0.70-1.21), p \text {-trend } 0.504, \text { soy } \\
\text { isoflavones } 0.67(0.53-0.86), \\
p \text {-trend } 0.001\end{array}$ & $\begin{array}{l}\text { age, sex, dialect group, } \\
\text { total energy intake, } \\
\text { smoking status, age of } \\
\text { smoking initiation, } \\
\text { amount smoked }\end{array}$ \\
\hline
\end{tabular}


Table 1. Cont.

\begin{tabular}{|c|c|c|c|c|c|c|c|c|c|}
\hline $\begin{array}{l}\text { Author, } \\
\text { Year, } \\
\text { Country, } \\
\text { Cohort }\end{array}$ & $\begin{array}{l}\text { Study } \\
\text { Design }\end{array}$ & Sample Size & Population & $\begin{array}{l}\text { Exposure } \\
\text { Assessment }\end{array}$ & $\begin{array}{l}\text { Outcome } \\
\text { Assessment }\end{array}$ & Follow-Up & $\begin{array}{l}\text { Statistical } \\
\text { Methods }\end{array}$ & Effect Measures & Covariates \\
\hline \multicolumn{10}{|c|}{ COPD and related symptoms } \\
\hline $\begin{array}{l}\text { Varraso et al., } \\
\text { 2010, US, } \\
\text { Nurses' Health } \\
\text { Study, Health } \\
\text { Professionals } \\
\text { Follow-up } \\
\text { Study [27] }\end{array}$ & C & 111,580 & $\begin{array}{c}\text { female nurses aged } \\
30-55 \text { years, men } \\
\text { health professionals } \\
40-75 \text { years, no } \\
\text { history of } \\
\text { asthma or COPD }\end{array}$ & $\begin{array}{c}\text { FFQs } \\
\text { administered in } \\
1984,1986,1990, \\
1994 \text { and 1998 in } \\
\text { NHS and in 1986, } \\
\begin{array}{c}1990 \text { and 1994 } \\
\text { in HPFS }\end{array}\end{array}$ & $\begin{array}{c}\text { self-reported } \\
\text { COPD defined by } \\
\text { doctor diagnosis } \\
\text { of chronic } \\
\text { bronchitis or } \\
\text { emphysema and } \\
\text { diagnostic test }\end{array}$ & $\begin{array}{l}16 \text { and } \\
12 \text { years }\end{array}$ & $\begin{array}{l}\text { Cox } \\
\text { proportional } \\
\text { hazard } \\
\text { regression } \\
\text { models }\end{array}$ & $\begin{array}{c}\text { total fibre } \\
\text { Q5 (28.4) vs. Q1 (11.2) } \\
\text { RR (95\% CI) } 0.67(0.50-0.90) \\
p \text {-trend } 0.03\end{array}$ & $\begin{array}{c}\text { age, sex, smoking status, } \\
\text { pack-years, pack-years2, } \\
\text { energy intake, BMI, US } \\
\text { region, physician visits, } \\
\text { physical activity, diabetes, } \\
\text { intakes of omega-3 (foods } \\
\text { and supplements), cured } \\
\text { meat, (glycaemic index, } \\
\text { carotenoids, } \\
\text { vitamins C, D, E) }\end{array}$ \\
\hline $\begin{array}{l}\text { Kaluza et al., } \\
\text { 2018, Sweden, } \\
\text { Cohort of } \\
\text { Swedish } \\
\text { Men [28] }\end{array}$ & C & 45,058 & $\begin{array}{l}\text { men aged } 45-79 \\
\text { years, no history } \\
\text { of COPD }\end{array}$ & 96-item FFQ & $\begin{array}{l}\text { incident COPD } \\
\text { cases through } \\
\text { linkage with } \\
\text { registry data }\end{array}$ & 13.1 years & $\begin{array}{l}\text { Cox } \\
\text { proportional } \\
\text { hazard } \\
\text { regression } \\
\text { models }\end{array}$ & $\begin{array}{c}\text { total fibre (g/day) } \\
\text { Q5 }(\geq 36.8) \text { vs. Q1 }(<23.7) \\
\text { HR }(95 \% \text { CI }) 0.62(0.53-0.71) \\
p \text {-trend }<0.001\end{array}$ & $\begin{array}{l}\text { age, education, BMI, total } \\
\text { physical activity, smoking } \\
\text { status, pack-years of } \\
\text { smoking, alcohol intake, } \\
\text { energy intake }\end{array}$ \\
\hline $\begin{array}{l}\text { Szmidt et al., } \\
\text { 2020, Sweden, } \\
\text { Swedish } \\
\text { Mammography } \\
\text { Cohort [29] }\end{array}$ & $\mathrm{C}$ & 35,339 & $\begin{array}{l}\text { women aged on } \\
\text { average } 62 \text { years, no } \\
\text { history of COPD }\end{array}$ & $\begin{array}{l}\text { 67-tem FFQ in } \\
\text { 1987, 96-item FFQ } \\
\text { in } 1997 \text { (baseline) }\end{array}$ & $\begin{array}{l}\text { incident COPD } \\
\text { cases through } \\
\text { linkage with } \\
\text { registry data }\end{array}$ & 11.5 years & $\begin{array}{l}\text { Cox } \\
\text { proportional } \\
\text { hazard } \\
\text { regression } \\
\text { models }\end{array}$ & $\begin{array}{c}\text { long-term total fibre (g/day) } \\
\text { Q5 ( } \geq 26.5) \text { vs. Q1 }(<17.6) \\
\text { HR (95\% CI) } 0.70(0.59-0.83) \\
p \text {-trend }<0.001\end{array}$ & $\begin{array}{c}\text { age, education, BMI, } \\
\text { walking/cycling, smoking } \\
\text { status, pack-years of } \\
\text { smoking, alcohol, } \\
\text { energy intake }\end{array}$ \\
\hline $\begin{array}{l}\text { Kim et al., 2019, } \\
\text { South Korea, } \\
\text { Korean } \\
\text { NHANES [30] }\end{array}$ & CS & 702 & $\begin{array}{l}\text { COPD adults } \\
\text { aged } \geq 40 \text { years }\end{array}$ & $24 \mathrm{~h}$ dietary recall & $\begin{array}{l}\text { COPD severity } \\
\text { defined by } \\
\text { spirometry }\end{array}$ & - & linear regression & $\begin{array}{c}\text { mean (SE) total fibre (g/day) } \\
\text { severity men } 20.9(1.7) \\
\text { women } 18.3(1.8)\end{array}$ & $\begin{array}{l}\text { sex, age, residential area, } \\
\text { educational level, } \\
\text { household income, } \\
\text { smoking status, height }\end{array}$ \\
\hline $\begin{array}{c}\text { Jung et al., 2021, } \\
\text { Korea [31] }\end{array}$ & $\mathrm{C}$ & 1439 & $\begin{array}{l}\text { adults aged on } \\
\text { average } 53 \text { years }\end{array}$ & $\begin{array}{l}117-\text { item FFQ } \\
\text { assessing intake } \\
\text { over the previous } \\
\text { three months, in } \\
2012 \text { and } 2017\end{array}$ & $\begin{array}{l}\text { incident COPD } \\
\text { cases defined by } \\
\text { spirometry }\end{array}$ & 5 years & $\begin{array}{l}\text { logistic } \\
\text { regression }\end{array}$ & $\begin{array}{c}\text { decrease in total fibre (g/day) } \\
\text { Q4 vs. Q1 decrease in total } \\
\text { fibre, proportion of new } \\
\text { airflow limitation cases } \\
5.85 \% \text { vs. } 1.39 \%\end{array}$ & $\begin{array}{l}\text { age, sex, smoking history, } \\
\text { baseline FEV1/FVC }\end{array}$ \\
\hline $\begin{array}{c}\text { Hanson et al., } \\
\text { 2016, US, } \\
\text { NHANES [32] }\end{array}$ & CS & 1921 & $\begin{array}{c}\text { adults aged } \\
40-79 \text { years (mean } \\
\text { age } 53 \text { years) }\end{array}$ & $\begin{array}{l}\text { two-interviewer } \\
\text { administered } \\
24 \mathrm{~h} \text { recalls }\end{array}$ & Lung function & - & $\begin{array}{l}\text { regression } \\
\text { analyses }\end{array}$ & $\begin{array}{c}\text { total fibre (g/day) } \\
\text { Q4 }(<10.75) \text { vs. Q1 ( }>17.5) \\
\text { Coefficients FEV1 } 82 \text { mL, FVC } \\
129 \text { mL, \% predicted FEV1 2.4, } \\
\% \text { predicted FVC } 2.8\end{array}$ & $\begin{array}{c}\text { age, sex, smoking status, } \\
\text { height, BMI, } \\
\text { socioeconomic status, total } \\
\text { energy intake, CRP, } \\
\text { vitamin E, a-carotene, } \\
\text { b-carotene, } \\
\text { b-cryptoxanthin, lycopene, } \\
\text { lutein plus zeaxanthin, } \\
\text { vitamin C and cured meat }\end{array}$ \\
\hline
\end{tabular}


Table 1. Cont.

\begin{tabular}{|c|c|c|c|c|c|c|c|c|c|}
\hline $\begin{array}{l}\text { Author, } \\
\text { Year, } \\
\text { Country, } \\
\text { Cohort }\end{array}$ & $\begin{array}{l}\text { Study } \\
\text { Design }\end{array}$ & Sample Size & Population & $\begin{array}{l}\text { Exposure } \\
\text { Assessment }\end{array}$ & $\begin{array}{l}\text { Outcome } \\
\text { Assessment }\end{array}$ & Follow-Up & $\begin{array}{l}\text { Statistical } \\
\text { Methods }\end{array}$ & Effect Measures & Covariates \\
\hline \multicolumn{10}{|c|}{ Lung function } \\
\hline $\begin{array}{l}\text { Root et al., 2014, } \\
\text { US, ARIC } \\
\text { study [33] }\end{array}$ & C & 12,532 & $\begin{array}{l}\text { adults aged on } \\
\text { average } 54 \text { years }\end{array}$ & $\begin{array}{c}\text { interviewer- } \\
\text { administered } \\
\text { semi- } \\
\text { quantitative FFQ }\end{array}$ & spirometry & 3 years & linear regression & $\begin{array}{c}\text { Coefficients per increase in one } \\
\text { quintile of total fibre FEV1 NS, } \\
\text { FVC NS, \%FEV1 } 0.201, \\
p \text {-trend } \leq 0.05, \text { FEV1/FVC } \\
0.129, p \text {-trend } \leq 0.01\end{array}$ & $\begin{array}{c}\text { age, sex, ethnicity, } \\
\text { education, total caloric } \\
\text { intake, physical activity, } \\
\text { current smoking, cigarette } \\
\text { years, height, BMI, and } \\
\text { interaction term black } \\
\text { ethnicity } \times \text { BMI }\end{array}$ \\
\hline $\begin{array}{l}\text { Lee et al., 2020, } \\
\text { Korea, } \\
\text { Ansan-Ansung } \\
\text { cohort [34] }\end{array}$ & C & 5880 & $\begin{array}{l}\text { non-COPD adults } \\
\text { median age } 50 \text { years }\end{array}$ & 103-item FFQ & $\begin{array}{c}\text { spirometry } \\
\text { (\% difference of } \\
\text { predicted FEV1 } \\
\text { between baseline } \\
\text { and follow-up) }\end{array}$ & 4 years & $\begin{array}{l}\text { logistic } \\
\text { regression }\end{array}$ & $\begin{array}{c}\mathrm{Q} 5(\geq 8.9) \text { vs. } \mathrm{Q} 1(\leq 4.4 \mathrm{~g} / \text { day }) \\
\text { decreased vs. } \\
\text { unchanged } / \text { improved } \\
\text { OR (95\% CI) } 0.83(0.61-1.12) \\
p \text {-trend } 0.080 \text { in men, } \\
1.14(0.86-1.51), p \text {-trend } 0.345 \\
\text { in women }\end{array}$ & $\begin{array}{l}\text { age, education, household } \\
\text { income, job, BMI, waist } \\
\text { circumference, } \\
\text { waist-to-hip ratio, } \\
\text { smoking, alcohol, exercise, } \\
\text { marriage status, history of } \\
\text { asthma and tuberculosis, } \\
\text { energy intake }\end{array}$ \\
\hline $\begin{array}{l}\text { Leng et al., 2017, } \\
\text { US, Lovelace } \\
\text { Smokers cohort } \\
\text { (LSC), Veteran } \\
\text { Smokers cohort } \\
\text { (VSC) [35] }\end{array}$ & C & $\begin{array}{l}1829 \text { in LSC, } \\
508 \text { in VSC }\end{array}$ & $\begin{array}{c}\text { adult smokers aged } \\
40-74 \text { years (mean } \\
\text { age } 57 \text { in LSC, } \\
62 \text { in VSC) }\end{array}$ & $\begin{array}{l}\text { semi-quantitative } \\
\text { 150-item FFQ }\end{array}$ & spirometry & 5.3 years & $\begin{array}{l}\text { linear mixed } \\
\text { effects model } \\
\text { with a } \\
\text { subject-specific } \\
\text { random } \\
\text { intercept, linear } \\
\text { regression }\end{array}$ & $\begin{array}{c}\text { total fibre (g/day), mean } 10.5 \\
\text { Coefficients (SE) FEV1 LSC } \\
80.9 \mathrm{~mL}(20.3), \\
\text { VSC } 97.8 \mathrm{~mL}(41.8), \\
\text { FEV1/FVC\% LSC 1.075 (0.403), } \\
\text { VSC } 2.018 \text { (0.761) }\end{array}$ & $\begin{array}{l}\text { age, sex, ethnicity, } \\
\text { smoking history, BMI, } \\
\text { educational level, height, } \\
\text { total caloric intake, time } \\
\text { since enrolment, baseline } \\
\text { FEV1 in decline analysis }\end{array}$ \\
\hline
\end{tabular}

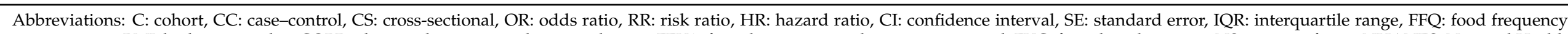

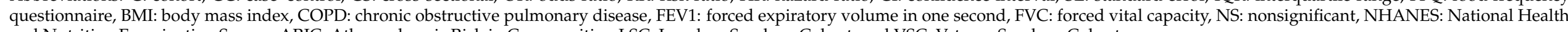
and Nutrition Examination Survey, ARIC: Atherosclerosis Risk in Communities, LSC: Lovelace Smokers Cohort, and VSC: Veteran Smokers Cohort. 


\subsection{Results of Individual Studies}

\subsubsection{Maternal Fibre Intake during Pregnancy}

An Australian cohort study of 639 mother-infant pairs, including infants with a family history of allergic disease, found that, although the total fibre intake during pregnancy was not associated with allergic disease in the offspring, a higher resistant starch intake was associated with a reduced risk of infant wheeze up to age 12 months (OR 0.68; 95\% CI 0.49-0.95) [16].

\subsubsection{Fibre Intake during Childhood}

Two cross-sectional studies reporting fibre intake during childhood were identified, with inconsistent results. An Australian study of 144 adolescents aged 12-18 years reported no association between fibre intake and self-reported wheeze [17]. However, a study among 4133 children aged 2-11 years from the US National Health and Nutrition Survey (NHANES) indicated that the odds of having asthma were higher for children who had a lower fibre intake (Q1 vs. Q4, ever asthma OR 1.31; 95\% CI 0.88-1.96, $p$-trend 0.034 and current asthma OR 1.38; 95\% CI 0.87-2.20, p-trend 0.027) [18]. In this study, the median fibre intake was $6.7 \mathrm{~g} / 1000 \mathrm{kcal}$, which is below the recommended US intake of $14 \mathrm{~g} / 1000 \mathrm{kcal}$.

\subsubsection{Fibre Intake during Adulthood}

Asthma, Rhinitis and Related Symptoms

Five studies reporting fibre intake during adulthood in relation to asthma, rhinitis and related symptoms were identified, with somewhat consistent results. In a cross-sectional study of 13,147 adults from the US NHANES, a low fibre intake was associated with increased odds of prevalent asthma (Q1 vs. Q4, OR 1.4; 95\% CI 1.0-1.8, $p$-trend 0.092), wheeze (OR 1.3; 95\% CI 1.0-1.6, $p$-trend 0.017), cough (OR 1.7; 95\% CI 1.2-2.3, $p$-trend $<0.001$ ) and phlegm (OR 1.4; 95\% CI 1.1-2.0, $p$-trend 0.011) [19]. Regarding asthma, stronger associations were seen for women and for non-Hispanic White adults. In a cross-sectional study of 10,479 adults from the Korean National Health and Nutrition Examination Survey (KNHANES), a higher dietary fibre intake was associated with reduced odds of asthma (Q4 vs. Q1, OR 0.66; 95\% CI 0.48-0.91, p-trend <0.001) and allergic rhinitis, the latter, however, only for Q2 vs. Q1 (OR 0.84; 95\% CI 0.70-1.00, $p$-trend < 0.001), especially in males [20]. In additional analyses, fibre intake reduced the allergic rhinitis symptoms, including watery rhinorrhoea and dog allergen sensitisation, only among males. However, in a cross-sectional study of 1002 Japanese pregnant women from the Osaka Maternal and Child Health Study, no association between fibre intake and allergic rhinitis was reported [21].

A French cross-sectional study of 26,640 women and 8740 men reported inverse associations between the highest quintile of total dietary fibre compared with the lowest quintile and the asthma symptom scores both among women (OR 0.73; 95\% CI 0.67-0.79, $p$-trend $<0.001$ ) and men (OR 0.63; 95\% CI 0.55-0.73, p-trend < 0.001) [22]. With regards to specific sources of fibre, the intake of fibre from cereals, fruit and seeds was most consistently associated with less asthma symptoms. Additionally, among participants with asthma, inverse associations were reported between the fibre intake and uncontrolled asthma. In an Australian case-control study of 137 participants with asthma and 65 healthy controls, participants with severe persistent asthma $(n=64)$ consumed, on average, $5 \mathrm{~g} /$ day less fibre as compared to healthy controls (OR 0.94; 95\% CI 0.90-0.99) [23].

\section{COPD and Related Symptoms}

Eight studies reporting the fibre intake in adulthood in relation to COPD and COPD symptoms were identified, with consistent results of a protective association. A crosssectional study of 11,897 participants of the Atherosclerosis Risk in Communities (ARIC) study in the US indicated a reduced prevalence of COPD with a higher total fibre intake (Q5 vs. Q1, OR 0.85; 95\% CI 0.68-1.05, $p$-trend $=0.044$ ). Inverse associations were also observed with cereal or fruit fibre but not with vegetable fibre [24]. No interaction with 
smoking status was observed, although associations were limited to current or ex-smokers. In a Japanese case-control study, high levels of total and insoluble dietary fibre were associated with a reduced risk of COPD (Q4 vs. Q1, OR 0.49; 95\% CI 0.26-0.95, p-trend 0.160 and OR $0.50 ; 95 \%$ CI $0.26-0.94$, $p$-trend 0.174 , respectively) [25].

A study of 49,140 cohort members from the Singapore Chinese Health Study examining the association between dietary fibre and new onset of cough with phlegm reported inverse associations with non-starch polysaccharides (Q4 vs. Q1, OR 0.61; 95\% CI 0.47-0.78, p-trend < 0.001), fruits (OR 0.67; 95\% CI 0.52-0.87, p-trend 0.006) and soy isoflavones (OR 0.67; 95\% CI 0.53-0.86, p-trend 0.001) [26]. Moreover, a large cohort study of 111,580 participants from the US Nurses' Health Study and Health Professionals Follow-up Study with long follow-up periods (16 and 12 years, respectively) reported inverse associations between the total dietary fibre intake and newly diagnosed COPD (Q5 vs. Q1, RR 0.67; 95\% CI 0.50-0.90, p-trend 0.03) [27]. Inverse associations were also observed with cereal fibre but not with fruit or vegetable fibre. In stratified analyses by the smoking status, associations were stronger among current smokers than among ex-smokers. Two cohort studies on dietary fibre intake from Sweden, which used registry data to identify incident COPD cases, confirmed the aforementioned results. The first study included 45,058 men from the Cohort of Swedish Men and reported strong inverse associations with the total fibre intake (Q5 vs. Q1, HR 0.62; 95\% CI 0.53-0.71, p-trend < 0.001), mainly in current smokers or ex-smokers but not in never smokers [28]. The second study included 35,339 women from the Swedish Mammography Cohort and evaluated the association between the baseline and long-term total fibre intake and COPD risk; in this study, a high long-term dietary fibre intake was associated with a reduced risk of COPD (Q5 vs. Q1, HR 0.70; 95\% CI 0.59-0.83, p-trend < 0.001), mainly in current or ex-smokers. For specific fibre sources, cereal and fruit fibre, but not vegetable fibre, were associated with a lower COPD risk [29].

A cross-sectional study of 702 adults with COPD from the KNHANES evaluated the association between disease severity and dietary nutrient intake; in this study, fibre intake was associated with a decreased severity of airway impairment in elderly men ( $\geq 60$ years old) with COPD but not in women [30]. Additionally, a cohort study of 1439 participants from Korea studied the relationship between new airflow limitation development, defined as FEV1/FVC < 0.70, and changes to the dietary pattern after a 5-year period; in this study, a $10 \%$ decreased intake of dietary fibre was associated with a newly developed airflow limitation (OR 2.71; 95\% CI 1.54-4.81) [31].

\section{Lung Function}

Six studies reporting fibre intake in adulthood in relation to lung function were identified, with generally consistent findings. The already mentioned study of 11,897 participants from the ARIC study in the US found positive cross-sectional associations between the total fibre and lung function (Q5 vs. Q1, forced expiratory volume in one sec (FEV1) $60.2 \mathrm{~mL}$; 95\% CI 27.7-92.7, p-trend < 0.001, forced vital capacity (FVC) $55.2 \mathrm{~mL}$; 95\% CI 18.2-92.3, p-trend 0.001 and FEV1/FVC 0.4; 95\% CI -0.1-0.9, p-trend 0.040). Similar patterns were seen for the fibre intake from cereal and fruit sources, while no association was observed for vegetable fibre [24]. Additionally, a recent cross-sectional study of 1921 participants from the US NHANES examined the association between fibre intake and measures of lung function. According to this study, a low fibre intake was associated with reduced measures of lung function (Q4 vs. Q1, FEV1 $82 \mathrm{~mL}(p=0.05)$, FVC $129 \mathrm{~mL}(p=0.01)$, \% predicted FEV1 2.4\% $(p=0.07)$ and \% predicted FVC 2.8\% $(p=0.02))$ [32].

Another prospective study of 12,532 adults from the ARIC study reported an increased fibre intake associated with improved lung function when followed up three years after the baseline; the coefficients per increase in one quintile of fibre intake were \%FEV1 0.201, $p$-trend $\leq 0.05$ and FEV1/FVC 0.129, $p$-trend $\leq 0.01$, but there was no association with FEV1 or FVC [33]. A prospective study including 5880 participants from the Korean AnsanAnsung cohort followed for four years indicated a positive association between the fibre 
intake and lung function among men but not among women [34]. A study among smokers in the US Lovelace Smokers cohort (LSC), with replication in the Veteran Smokers cohort (VSC), identified, among other nutrients, the fibre intake to be significantly associated with a better average FEV1 (LSC $80.9 \mathrm{~mL}$; SE 20.3, $p=0.0032$ and VSC $97.8 \mathrm{~mL}$; SE 41.8, $p=0.045$ ) [35]. Finally, in cross-sectional analyses in the aforementioned Australian casecontrol study of 137 participants with asthma and 65 healthy controls, the fibre intake was positively associated with FEV1, FVC and FEV1/FVC (coefficient per unit increase in fibre intake $0.02 \mathrm{~L}(p=0.001), 0.02 \mathrm{~L}(p=0.002)$ and $0.2 \%(p=0.035)$, respectively) and negatively associated with airway eosinophilia $(-0.36 \%(p=0.005))$ among participants with asthma [23].

\subsection{Quality of Studies}

\subsubsection{Asthma and Related Symptoms}

Out of seven studies, five were cross-sectional [17-20,22], one was a case-control [23] and one a cohort study [16]. With regards to fibre intake assessment, four studies used food frequency questionnaires (FFQ) $[16,17,20,23]$, and three studies used 24-h dietary recalls $[18,19,22]$, including repeated assessments in the last two studies. Regarding the outcome assessment, asthma was self-reported in all studies, while five studies also included a clinical examination with spirometry, skin prick tests and/or blood sampling $[16,17,19,20,23]$. All studies adjusted for age and sex, while most studies adjusted for body mass index (BMI) or energy intake, socioeconomic factors and smoking. Overall, the articles were assigned a moderate-to-serious risk of bias (Figure 2a).

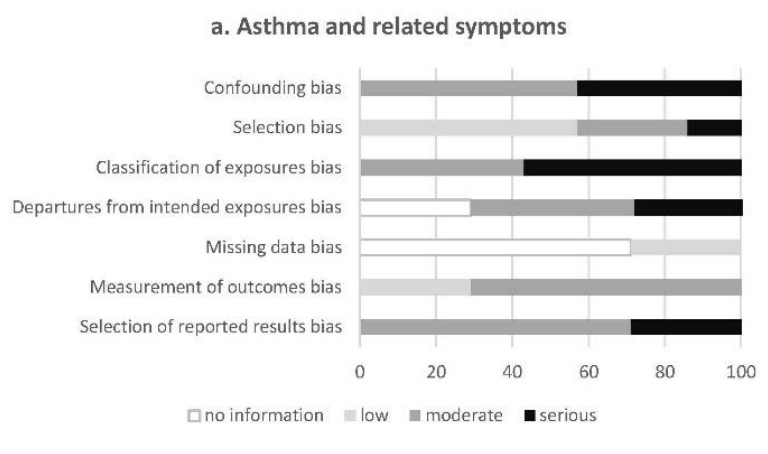

c. COPD and related symptoms

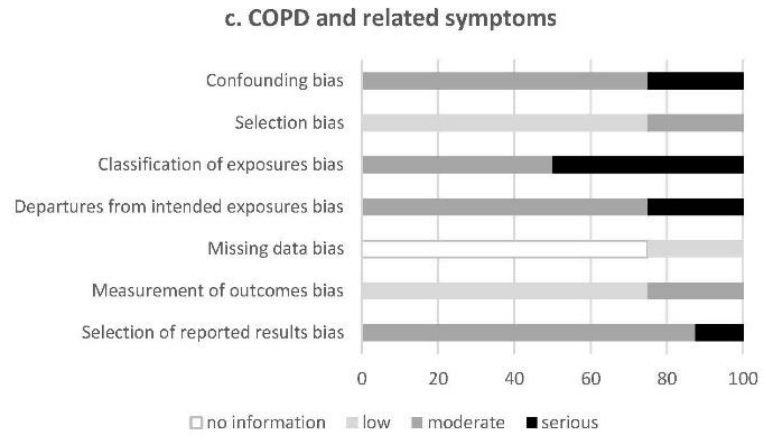

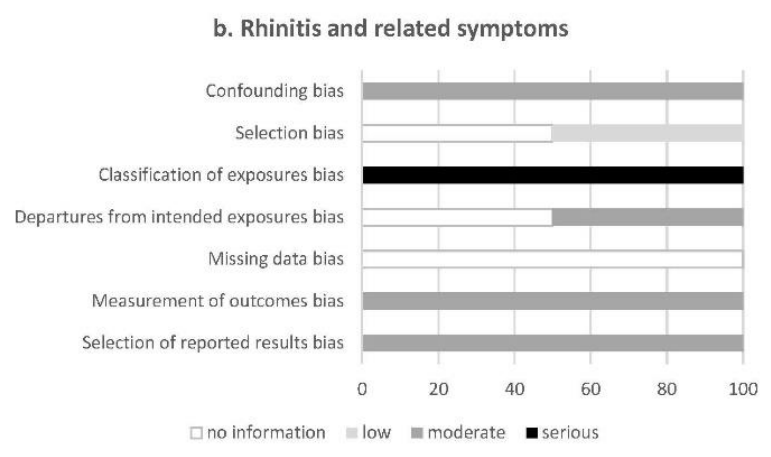

d. Lung function

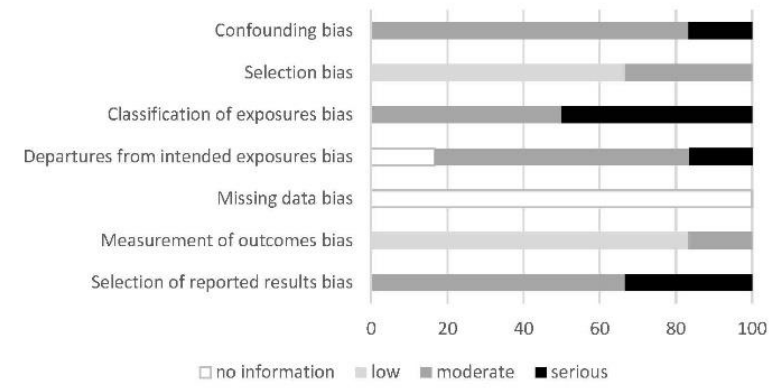

Figure 2. Risk of bias in individual studies, showing the proportion of studies with no information and low, moderate and serious risks of bias in each domain of the RoB-NObs tool.

\subsubsection{Rhinitis and Related Symptoms}

Both studies had a cross-sectional study design [20,21]. To assess the fibre intake, both studies used a FFQ $[20,21]$. Allergic rhinitis was self-reported in both studies and assessed based on the symptoms and, additionally, nasal endoscopy and serum IgE levels in one study [20], while the assessment was based on drug treatment in the previous 12 months in the other study [21]. Both studies adjusted for major potential confounders, 
including age, BMI, socioeconomic factors and smoking. Overall, the articles were assigned a moderate-to-serious risk of bias (Figure $2 b$ ).

\subsubsection{COPD and Related Symptoms}

Out of eight studies, five were cohorts [26-29,31], two were cross-sectional $[24,30]$ and one a case-control study [25]. Fibre intake was assessed using FFQs in all but one study [24-29,31], with repeated assessments in three studies [27,29,31] and one study using a 24-h dietary recall [30]. COPD and related symptoms were assessed using selfreported questionnaires in three studies $[24,26,27]$, spirometry in four studies $[24,25,30,31]$ and registries in two studies $[28,29]$ (one study used both self-reported and spirometry diagnosed definitions). All the studies adjusted for major potential confounders, including age, sex and smoking, and most adjusted for BMI or energy intake and socioeconomic factors, while some additionally adjusted for lifestyle (physical activity and alcohol intake) and other dietary factors. Overall, the articles were assigned a moderate risk of bias (Figure 2c).

\subsubsection{Lung Function}

Out of six studies, there were three cohort [33-35] and three cross-sectional studies [23,24,32]. Fibre intake was assessed using FFQs in all studies, apart from one study that used repeated 24-h dietary recalls [32]. Lung function was measured by spirometry in all the studies and additionally using eNO and combined bronchial provocation and sputum induction in one study [23]. Most of the studies adjusted for major potential confounders, including age, sex, BMI, total energy intake, smoking and socioeconomic factors. Overall, the articles were assigned a moderate risk of bias (Figure 2d).

\subsection{Strength of the Evidence}

In the present study, we did not include intervention studies in the eligibility criteria. According to the WCRF criteria, and based on the available evidence from observational studies, the overall strength of the evidence was graded as limited/no conclusion (insufficient) with regards to asthma and rhinitis, probable (moderate) for COPD and limited/suggestive (low) for lung function.

\section{Discussion}

This review sought to explore if there is a protective association between dietary fibre intake and asthma, rhinitis, COPD and lung function and, if so, which sources of fibre are the most beneficial. The findings show that the current evidence from observational studies is limited and inconclusive with regards to asthma and rhinitis. There is suggestive evidence that dietary fibres may be associated with improved lung function in the general adult population, with very few studies reporting fibre intake in high-risk populations. Moreover, there is probable evidence for a beneficial role of fibres in the risk of COPD, which is considered as strong evidence according to the WCRF criteria.

Based on the intake level observed to protect against coronary heart disease, an adequate intake of total fibre has been set to 30-35 g/day and 25-32 g/day for adult men and women, respectively [36], and 10-40 $\mathrm{g}$ for children and adolescents, depending on age, gender and energy intake [37]. The mean fibre intake was reported to be below the recommended levels in all the included studies, and only subjects in the highest quartile/quintile of fibre intake met the recommendations. Additionally, geographical differences in the amount of total dietary fibre intake were observed, with lower intakes reported in studies from countries in Asia, followed by the US, and higher intakes in studies from countries in Australia and Europe. With regards to different types of diets, while the fibre content of animal products is scarce, plant-based diets include fibre-rich foods, such as cereals, fruits, vegetables and nuts, in abundance. We observed a difference in the sources of dietary fibres in the studied populations as well, reflecting different dietary patterns; however, this was not consistently reported in all the included studies. The 
current evidence is not conclusive about which fibre-rich foods are most beneficial for respiratory health; the grain sources of dietary fibre have been shown to be more beneficial compared to fruits and vegetables, but it is unclear if this is due to their higher fibre content, greater amounts consumed, less probability of measurement error, displacement of high-energy foods and overall diet quality or associated lifestyle factors, such as greater levels of physical activity [36].

We were able to identify only one study on maternal fibre intake during pregnancy in relation to allergic disease in the offspring. Although these results do not support an association between prenatal exposure to dietary fibre and allergic disease, the association is biologically plausible. In two recent birth cohort studies, the faecal concentration of SCFAs during pregnancy was inversely associated with asthma and allergic rhinitis in the offspring up to 6 years [38,39]. In one of the studies, which was part of a randomised controlled trial and, therefore, not included in the selection criteria of our review, the fibre intake in pregnancy was positively associated with the total SCFAs but not with any of the atopic outcomes in the offspring [38]. It was therefore hypothesised that dietary fibres contribute to offspring disease risk only in combination with the relevant intestinal microbes. These findings, supported by studies in animal models $[39,40]$, require further replication in observational studies with a larger sample size and can potentially pave the way to microbiome-targeted interventions to prevent asthma and atopy in the offspring [9,41,42].

Additionally, the two selected studies on dietary fibre intake during childhood showed inconsistent results. However, previous reviews have reported protective associations between fruit and vegetable consumption, or dietary patterns rich in fruits, vegetables, legumes and cereals (such as the Mediterranean diet), and asthma or wheeze among children [43-45]. Moreover, a protective association between whole grains and asthma among children has been reported [46]. These associations may be partly explained by the concomitant intake of dietary fibres. Dietary fibres can potentially improve airway inflammation by promoting anti-inflammatory cytokines, improving glucose control, and modulating the gut immunologic response [10]. On the other hand, asthma is a heterogeneous disease, and asthma development is a dynamic process, characterised by remission, relapse and a new onset of symptoms from childhood up to adulthood [47]. A reduced fibre intake has been observed among adults with severe asthma and has been associated with increased eosinophilic airway inflammation [23]. Lung function growth may not only be impaired during early childhood but also continues throughout adolescence and early adulthood [48]. Thus, a critical period of development is missed by the current body of evidence, addressing dietary fibre intake and asthma and lung function impairment in adult populations. A paucity of studies addressing asthma severity, different asthma phenotypes and lung function among participants with asthma has also been identified.

We were able to identify only two studies on fibre intake in relation to allergic rhinitis, with inconsistent results, and no study on nonallergic rhinitis. Allergic rhinitis is associated with sensitisation to inhalant allergens, whereas nonallergic rhinitis is a nasal mucosal inflammation without systemic signs of allergic inflammation, associated with exposure to irritants, hormonal dysfunction and specific medications [49,50]. Regarding allergic rhinitis, a high fibre intake in a murine model showed less eosinophil infiltration, less goblet cell metaplasia in the nasal mucosa and decreased Th2 cytokines compared to a low intake [51]. In a study among children, adherence to the Mediterranean diet has been inversely associated with allergic rhinitis [52]. Further research is needed on dietary fibre intake and rhinitis outcomes, both among children and adults.

In our review, we identified eight studies on fibre intake in relation to COPD and related symptoms reporting consistent results of a protective association, which has also been suggested by two previous systematic reviews of studies on fibre intake in relation to COPD, partly based on the same studies [53,54]. The association with COPD may be explained by the antioxidant and anti-inflammatory properties of dietary fibres, including lower levels of C-reactive protein and proinflammatory cytokines and higher levels of some anti-inflammatory cytokines, such as adiponectin [54]. In addition, high dietary 
fibre has been suggested to attenuate innate immune-mediated systemic and pulmonary inflammation through the presence of a gut-liver-lung axis [55]. The stronger inverse association with COPD among current or ex-smokers may be explained by the higher oxidative stress in these groups, as well as the continued endogenous production of reactive oxygen species even after smoking cessation. Among non-smokers, the mechanisms related to COPD development may differ from those in current or ex-smokers and relate more to genetic predisposition and environmental exposures [28]. Among smokers, lung function was improved via the increased intake of dietary fibre, further supporting the importance of the gut-liver-lung axis in COPD [41]. On the other hand, a protective effect of fibre intake on lung function in both smokers and non-smokers has also been observed [24]. In nonsmokers, fibre intake may protect against the deleterious effects of indoor and ambient air pollutants. Considering the sources of dietary fibres, the results from the included studies suggest that fibres from cereals and fruits, but not vegetables, are inversely associated with the risk of COPD. It has been suggested that similar protective associations of a higher intake of cereal fibre and of the total dietary fibre may be because of the high dietary fibre content of cereals [56]. Nevertheless, the lack of an inverse association with vegetables has been suggested to be related to the higher uptake of heavy metals, especially cadmium and lead, from vegetables compared to fruits [29].

The strength of evidence is primarily related to the methodological quality of the included studies. Most of the studies used questionnaires to assess dietary fibre intake, which might have led to some misclassification of the exposure, however nondifferential with regards to the outcomes of interest. Although the absolute fibre intake may be difficult to be estimated by FFQs, the ranking of participant intakes is possible and sufficient in this type of analytic epidemiologic studies [57]. In studies where multiple exposures were studied or fibre was part of an overall dietary pattern, the methods used to assess the exposure were less well-described, which hampers systematic reviews and metaanalyses [58]. In line with this, an increased risk of selective reporting could be inferred from studies reporting associations with multiple outcomes and analyses among different subgroups [13]. The risk of publication bias for studies finding no associations between fibre intake and respiratory and atopic outcomes should also be considered. Although most of the studies extensively adjusted their analyses for major potential confounders and some additionally included dietary and lifestyle factors, such as smoking, physical activity and alcohol consumption, residual confounding cannot be completely ruled out. In this systematic review, we were able to assess the strength of the current evidence based on observational studies and highlight specific areas where further research is needed.

\section{Conclusions}

In conclusion, the current evidence from observational studies on dietary fibre intake is probable (moderate) for an inverse association with COPD and limited/suggestive (low) for an association with lung function in the general adult population. In contrast, there is insufficient evidence for an association with asthma or rhinitis in adults. Thus, further research is needed with regards to asthma, rhinitis and lung function in adults, as well as among children.

Supplementary Materials: The following are available online at https:/ /www.mdpi.com/article/10 .3390/nu13103594/s1: Table S1: Completed PRISMA checklist, Table S2: Medline search construct and Table S3: Documentation of the search strategies.

Author Contributions: Conceptualisation, E.S. and A.B.; methodology, E.S.; software, E.S. and A.V.G.; validation, S.E.; formal analysis, E.S. and A.V.G.; investigation, E.S. and A.V.G.; writingoriginal draft preparation, E.S.; writing-review and editing, S.E., A.V.G. and A.B.; visualisation, E.S.; supervision, A.B.; project administration, E.S. and funding acquisition, A.B. All authors have read and agreed to the published version of the manuscript.

Funding: This research was funded by Forte (Swedish Research Council for health, working life and welfare), project number 2017-00526. 
Institutional Review Board Statement: Not applicable.

Informed Consent Statement: Not applicable.

Data Availability Statement: Data is contained within the article or supplementary material.

Acknowledgments: The authors would like to thank the librarians at the Karolinska Institute University Library who were involved in the process of searching the literature prior to the systematic review.

Conflicts of Interest: The authors declare no conflict of interest. The funders had no role in the design of the study; in the collection, analyses or interpretation of the data; in the writing of the manuscript or in the decision to publish the results.

\section{References}

1. Veronese, N.; Solmi, M.; Caruso, M.G.; Giannelli, G.; Osella, A.R.; Evangelou, E.; Maggi, S.; Fontana, L.; Stubbs, B.; Tzoulaki, I. Dietary fiber and health outcomes: An umbrella review of systematic reviews and meta-analyses. Am. J. Clin. Nutr. 2018, 107, 436-444. [CrossRef]

2. Park, Y.; Subar, A.; Hollenbeck, A.; Schatzkin, A. Dietary fiber intake and mortality in the NIH-AARP diet and health study. Arch. Intern. Med. 2011, 171, 1061-1068. [CrossRef]

3. Chuang, S.-C.; Norat, T.; Murphy, N.; Olsen, A.; Tjonneland, A.; Overvad, K.; Boutron-Ruault, M.C.; Perquier, F.; Dartois, L.; Kaaks, R.; et al. Fiber intake and total and cause-specific mortality in the European Prospective Investigation into Cancer and Nutrition cohort. Am. J. Clin. Nutr. 2012, 96, 164-174. [CrossRef]

4. Dreher, M. Whole Fruits and Fruit Fiber Emerging Health Effects. Nutrients 2018, 10, 1833. [CrossRef]

5. Global Asthma Network. The Global Asthma Report; Auckland, New Zealand, 2018. Available online: http:/ / globalasthmareport. org / (accessed on 8 September 2021).

6. Melén, E.; Guerra, S.; Hallberg, J.; Jarvis, D.; Stanojevic, S. Linking COPD epidemiology with pediatric asthma care: Implications for the patient and the physician. Pediatr. Allergy Immunol. 2019, 30, 589-597. [CrossRef]

7. Ballardini, N.; Kull, I.; Lind, T.; Hallner, E.; Almqvist, C.; Ostblom, E.; Melén, E.; Pershagen, G.; Lilja, G.; Bergström, A.; et al. Development and comorbidity of eczema, asthma and rhinitis to age 12: Data from the BAMSE birth cohort. Allergy 2012, 67, 537-544. [CrossRef]

8. Gibson, G.; Loddenkemper, R.; Sibille, Y.; Lundbäck, B. Childhood Asthma. European Lung White Book, 2nd ed.; European Respiratory Society: Sheffield, UK, 2013; pp. 126-137.

9. McKenzie, C.; Tan, J.; Macia, L.; Mackay, C.R. The nutrition-gut microbiome-physiology axis and allergic diseases. Immunol. Rev. 2017, 278, 277-295. [CrossRef]

10. Alwarith, J.; Kahleova, H.; Crosby, L.; Brooks, A.; Brandon, L.; Levin, S.M.; Barnard, N.D. The role of nutrition in asthma prevention and treatment. Nutr. Rev. 2020, 78, 928-938. [CrossRef]

11. Palafox-Carlos, H.; Ayala-Zavala, J.; González-Aguilar, G. The role of dietary fiber in the bioaccessibility and bioavailability of fruit and vegetable antioxidants. J. Food Sci. 2011, 76, R6-R15. [CrossRef]

12. Page, M.J.; McKenzie, J.E.; Bossuyt, P.M.; Boutron, I.; Hoffmann, T.C.; Mulrow, C.D.; Shamseer, L.; Tetzlaff, J.M.; Akl, E.A.; Brennan, S.E.; et al. The PRISMA 2020 statement: An updated guideline for reporting systematic reviews. BMJ $2021,372 n 71$. [CrossRef]

13. Nutrition Evidence Systematic Review. Risk of Bias for Nutrition Observational Studies (RoB-NObs) Tool. 2019. Available online: https: / nesr.usda.gov/sites / default/files / 2019-07/RiskOfBiasForNutritionObservationalStudies-RoB-NObs.pdf (accessed on 8 September 2021).

14. World Cancer Research Fund, American Institute for Cancer Research. Judging the evidence. In Continuous Update Project Report; World Cancer Research Fund: London, UK, 2018.

15. Arnesen, E.K.; Christensen, J.J.; Andersen, R.; Eneroth, H.; Erkkola, M.; Høyer, A.; Lemming, E.W.; Meltzer, H.M.; Pórhallsson, I.; Pórsdóttir, I.; et al. The Nordic Nutrition Recommendations 2022-Handbook for qualified systematic reviews. Food Nutr. Res. 2020, 64. [CrossRef]

16. Pretorius, R.A.; Bodinier, M.; Prescott, S.L.; Palmer, D.J. Maternal Fiber Dietary Intakes during Pregnancy and Infant Allergic Disease. Nutrients 2019, 11, 1767. [CrossRef]

17. Wood, L.G.; Lagleva, M.; Shah, S.; Berthon, B.S.; Galbraith, S.; Henry, R.; Kepreotes, H.; Gibson, P.G. Dietary changes in migrant adolescents with increasing length of stay in Australia and associated risk of wheeze-A retrospective, cross sectional study. BMC Pediatr. 2015, 15, 102. [CrossRef]

18. Vaccaro, J.A.; Niego, J.; Huffman, F.G. Dietary factors, body weight, and screen time in U.S. children with and without asthma. J. Child. Health Care 2016, 45, 22-38. [CrossRef]

19. Saeed, M.A.; Gribben, K.C.; Alam, M.; Lyden, E.R.; Hanson, C.K.; LeVan, T.D. Association of Dietary Fiber on Asthma, Respiratory Symptoms, and Inflammation in the Adult National Health and Nutrition Examination Survey Population. Ann. Am. Thorac. Soc. 2020, 17, 1062-1068. [CrossRef]

20. Lee, H.; Lee, K.; Son, S.; Kim, Y.-C.; Kwak, J.; Kim, H.; Lee, S.; Kim, T. Association of allergic diseases and related conditions with dietary fiber intake in Korean adults. Int. J. Environ. Res. Public Health 2021, 18, 1-10. 
21. Miyake, Y.; Sasaki, S.; Ohya, Y.; Miyamoto, S.; Matsunaga, I.; Yoshida, T.; Hirota, Y.; Oda, H. Dietary intake of seaweed and minerals and prevalence of allergic rhinitis in Japanese pregnant females: Baseline data from the Osaka Maternal and Child Health Study. Ann. Epidemiol. 2006, 16, 614-621. [CrossRef]

22. Andrianasolo, R.M.; Hercberg, S.; Kesse-Guyot, E.; Druesne-Pecollo, N.; Touvier, M.; Galan, P.; Varraso, R. Association between dietary fibre intake and asthma (symptoms and control): Results from the French national e-cohort NutriNet-Sante. Br. J. Nutr. 2019, 122, 1040-1051. [CrossRef]

23. Berthon, B.S.; Macdonald-Wicks, L.K.; Gibson, P.G.; Wood, L.G. Investigation of the association between dietary intake, disease severity and airway inflammation in asthma. Respirology 2013, 18, 447-454. [CrossRef]

24. Kan, H.; Stevens, J.; Heiss, G.; Rose, K.M.; London, S.J. Dietary fiber, lung function, and chronic obstructive pulmonary disease in the atherosclerosis risk in communities study. Am. J. Epidemiol. 2008, 167, 570-578. [CrossRef]

25. Hirayama, F.; Lee, A.H.; Binns, C.; Zhao, Y.; Hiramatsu, T.; Tanikawa, Y.; Nishimura, K.; Taniguchi, H. Do vegetables and fruits reduce the risk of chronic obstructive pulmonary disease? A case-control study in Japan. Prev. Med. 2009, 49, 184-189. [CrossRef]

26. Butler, L.M.; Koh, W.-P.; Lee, H.-P.; Yu, M.C.; London, S.J. Dietary fiber and reduced cough with phlegm: A cohort study in Singapore. Am. J. Respir. Crit. Care Med. 2004, 170, 279-287. [CrossRef]

27. Varraso, R.; Willett, W.C.; Camargo, C.A., Jr. Prospective study of dietary fiber and risk of chronic obstructive pulmonary disease among US women and men. Am. J. Epidemiol. 2010, 171, 776-784. [CrossRef]

28. Kaluza, J.; Harris, H.; Wallin, A.; Linden, A.; Wolk, A. Dietary Fiber Intake and Risk of Chronic Obstructive Pulmonary Disease: A Prospective Cohort Study of Men. Epidemiology 2018, 29, 254-260. [CrossRef]

29. Szmidt, M.K.; Kaluza, J.; Harris, H.R.; Linden, A.; Wolk, A. Long-term dietary fiber intake and risk of chronic obstructive pulmonary disease: A prospective cohort study of women. Eur. J. Nutr. 2020, 59, 1869-1879. [CrossRef]

30. Kim, T.; Choi, H.; Kim, J. Association between Dietary Nutrient Intake and Chronic Obstructive Pulmonary Disease Severity: A Nationwide Population-Based Representative Sample. COPD 2020, 17, 49-58. [CrossRef]

31. Jung, Y.J.; Lee, S.H.; Chang, J.H.; Lee, H.S.; Kang, E.H.; Lee, S.W. The Impact of Changes in the Intake of Fiber and Antioxidants on the Development of Chronic Obstructive Pulmonary Disease. Nutrients 2021, 13, 580. [CrossRef]

32. Hanson, C.; Lyden, E.; Rennard, S.; Mannino, D.M.; Rutten, E.P.A.; Hopkins, R.; Young, R. The Relationship between Dietary Fiber Intake and Lung Function in the National Health and Nutrition Examination Surveys. Ann. Am. Thorac. Soc. 2016, 13, 643-650. [CrossRef]

33. Root, M.M.; Houser, S.M.; Anderson, J.J.B.; Dawson, H.R. Healthy Eating Index 2005 and selected macronutrients are correlated with improved lung function in humans. Nutr. Res. 2014, 34, 277-284. [CrossRef]

34. Lee, S.-A.; Joshi, P.; Kim, Y.; Kang, D.; Kim, W.J. The Association of Dietary Macronutrients with Lung Function in Healthy Adults Using the Ansan-Ansung Cohort Study. Nutrients 2020, 12, 2688. [CrossRef]

35. Leng, S.; Picchi, M.A.; Tesfaigzi, Y.; Wu, G.; Gauderman, W.J.; Xu, F.; Gilliland, F.D.; Belinsky, S.A. Dietary nutrients associated with preservation of lung function in Hispanic and non-Hispanic white smokers from New Mexico. Int. J. Chronic Obstr. Pulm. Dis. 2017, 12, 3171-3181. [CrossRef] [PubMed]

36. Stephen, A.M.; Champ, M.M.-J.; Cloran, S.J.; Fleith, M.; Van Lieshout, L.; Mejborn, H.; Burley, V.J. Dietary fibre in Europe: Current state of knowledge on definitions, sources, recommendations, intakes and relationships to health. Nutr. Res. Rev. 2017, 30, 149-190. [CrossRef]

37. Edwards, C.; Xie, C.; Garcia, A. Dietary fibre and health in children and adolescents. Proc. Nutr. Soc. 2015, 73, 292-302. [CrossRef]

38. Lee-Sarwar, K.A.; Kelly, R.S.; Lasky-Su, J.; Zeiger, R.S.; O'Connor, G.T.; Sandel, M.T.; Bacharier, L.B.; Beigelman, A.; Rifas-Shiman, S.L.; Carey, V.J.; et al. Fecal short-chain fatty acids in pregnancy and offspring asthma and allergic outcomes. J. Allergy Clin. Immunol. Pract. 2020, 8, 1100-1102.e13. [CrossRef] [PubMed]

39. Roduit, C.; Frei, R.; Ferstl, R.; Loeliger, S.; Westermann, P.; Rhyner, C.; Schiavi, E.; Barcik, W.; Rodriguez-Perez, N.; Wawrzyniak, M.; et al. High levels of Butyrate and Propionate in early life are associated with protection against atopy. Allergy Eur. J. Allergy Clin. Immunol. 2018, 74, 799-809. [CrossRef] [PubMed]

40. Thorburn, A.N.; McKenzie, C.I.; Shen, S.; Stanley, D.; Macia, L.; Mason, L.J.; Roberts, L.K.; Wong, C.; Shim, R.; Robert, R.; et al. Evidence that asthma is a developmental origin disease influenced by maternal diet and bacterial metabolites. Nat. Commun. 2015, 6, 7320. [CrossRef]

41. Zhang, D.P.; Li, S.; Wang, N.; Tan, H.Y.; Zhang, Z.M.; Feng, Y.B. The Cross-Talk Between Gut Microbiota and Lungs in Common Lung Diseases. Front. Microbiol. 2020, 11, 301. [CrossRef] [PubMed]

42. Vuillermin, P.J.; Macia, L.; Nanan, R.; Tang, M.L.K.; Collier, F.; Brix, S. The maternal microbiome during pregnancy and allergic disease in the offspring. Semin. Immunopathol. 2017, 39, 669-675. [CrossRef]

43. Seyedrezazadeh, E.; Moghaddam, M.; Ansarin, K.; Vafa, M.; Sharma, S.; Kolahdooz, F. Fruit and vegetable intake and risk of wheezing and asthma: A systematic review and meta-analysis. Nutr. Rev. 2014, 72, 411-428. [CrossRef]

44. Hosseini, B.; Berthon, B.; Wark, P.; Wood, L. Effects of Fruit and Vegetable Consumption on Risk of Asthma, Wheezing and Immune Responses: A Systematic Review and Meta-Analysis. Nutrients 2017, 9, 341. [CrossRef]

45. Zhang, Y.; Lin, J.; Fu, W.; Liu, S.; Gong, C.; Dai, J. Mediterranean diet during pregnancy and childhood for asthma in children: A systematic review and meta-analysis of observational studies. Pediatr. Pulmonol. 2019, 54, 949-961. [CrossRef] [PubMed]

46. Tabak, C.; Wijga, A.; de Meer, G.; Janssen, N.; Brunekreef, B.; Smit, H. Diet and asthma in Dutch school children (ISAAC-2). Thorax 2006, 61, 1048-1053. [CrossRef] [PubMed] 
47. Fuchs, O.; Bahmer, T.; Rabe, K.; von Mutius, E. Asthma transition from childhood into adulthood. Lancet Respir. Med. 2017, 5, 224-234. [CrossRef]

48. Koefoed, H.; Zwitserloot, A.; Vonk, J.; Koppelman, G. Asthma, bronchial hyperresponsiveness, allergy and lung function development until early adulthood: A systematic literature review. Pediatr. Allergy Immunol. 2021, 32, 1238-1254. [CrossRef]

49. Roberts, G.; Xatzipsalti, M.; Borrego, L.M.; Custovic, A.; Halken, S.; Hellings, P.; Papadopoulos, N.; Rotiroti, G.; Scadding, G.; Timmermans, F.; et al. Paediatric rhinitis: Position paper of the European Academy of Allergy and Clinical Immunology. Allergy 2013, 68, 1102-1116. [CrossRef] [PubMed]

50. Hellings, P.W.; Klimek, L.; Cingi, C.; Agache, I.; Akdis, C.; Bachert, C.; Bousquet, J.; Demoly, P.; Gevaert, P.; Hox, V.; et al. Non-allergic rhinitis: Position paper of the European Academy of Allergy and Clinical Immunology. Allergy 2017, 72, 1657-1665. [CrossRef]

51. Zhang, Z.Y.; Shi, L.; Pang, W.H.; Liu, W.W.; Li, J.F.; Wang, H.B.; Shi, G. Dietary Fiber Intake Regulates Intestinal Microflora and Inhibits Ovalbumin-Induced Allergic Airway Inflammation in a Mouse Model. PLoS ONE 2016, 11. [CrossRef] [PubMed]

52. Chatzi, L.; Apostolaki, G.; Bibakis, I.; Skypala, I.; Bibaki-Liakou, V.; Tzanakis, N.; Kogevinas, M.; Cullinan, P. Protective effect of fruits, vegetables and the Mediterranean diet on asthma and allergies among children in Crete. Thorax 2007, 62, 677-683. [CrossRef]

53. Fonseca Wald, E.L.A.; van den Borst, B.; Gosker, H.R.; Schols, A.M.W.J. Dietary fibre and fatty acids in chronic obstructive pulmonary disease risk and progression: A systematic review. Respirology 2014, 19, 176-184. [CrossRef] [PubMed]

54. Seyedrezazadeh, E.; Pour Moghaddam, M.; Ansarin, K.; Jafarabadi, M.A.; Sharifi, A.; Sharma, S.; Kolahdooz, F. Dietary Factors and Risk of Chronic Obstructive Pulmonary Disease: A Systemic Review and Meta-Analysis. Tanaffos 2019, 18, $294-309$.

55. Young, R.P.; Hopkins, R.J.; Marsland, B. The Gut-Liver-Lung Axis. Modulation of the Innate Immune Response and Its Possible Role in Chronic Obstructive Pulmonary Disease. Am. J. Respir. Cell Mol. Biol. 2016, 54, 161-169. [CrossRef]

56. Reynolds, A.; Mann, J.; Cummings, J.; Winter, N.; Mete, E.; Te Morenga, L. Carbohydrate quality and human health: A series of systematic reviews and meta-analyses. Lancet 2019, 393, 434-445. [CrossRef]

57. Willett, W.C. Nutritional Epidemiology, 3rd ed.; Oxford University Press: Oxford UK, 2013.

58. Lachat, C.; Hawwash, D.; Ocké, M.; Berg, C.; Forsum, E.; Hörnell, A.; Larsson, C.; Sonestedt, E.; Wirfält, E.; Åkesson, A.; et al. Strengthening the Reporting of Observational Studies in Epidemiology-Nutritional Epidemiology (STROBE-nut): An Extension of the STROBE Statement. PLoS Med. 2016, 13, e1002036. [CrossRef] 